\title{
Determination of anisotropic properties of carbon fiber composites for civil engineering applications using infrared thermography with periodic excitation
}

\author{
by L. Ibos*, J. Dumoulin** and V. Feuillet*
}

* Univ. Paris-Est, CERTES/OSU Efluve, F-94010 Créteil, France, ibos@u-pec.fr, vincent.feuillet@u-pec.fr

** LUNAM Université, IFSTTAR, COSYS/SII, F-44340, Bouguenais, France, jean.dumoulin@ifsttar.fr

\begin{abstract}
This papers presents the development of a new device for the determination of thermal conductivity and diffusivity of anisotropic composite plates. The excitation signal is provided through a thermoelectric cooler and does not require any optical source like a laser source for instance. Infrared thermography is used to follow apparent surface temperature evolution with time. Experiments were carried out two composite sample systems (with two different fiber orientations). Result analysis is presented and discussed.
\end{abstract}

\section{Introduction}

Carbon fiber composites are extensively used in many engineering fields, e.g. for the reinforcement of civil engineering structures [1]. These materials have highly anisotropic properties due to the fact that carbon fibers are generally oriented in the plane of composites plates or tissues and can be glued with by using thermosettings or thermoplastics binders. Active infrared thermography is already used for in-situ non-destructive testing of composites [23]. Thus, thermophysical properties of these materials are to be known precisely, for instance to estimate properties of defects detected by an IR thermography inspection [4]. The paper deals with the development of a new set-up to determine anisotropic thermal properties of this kind of material, particularly of longitudinal thermal diffusivity and conductivity.

\section{Materials and Methods}

\subsection{Samples investigated}

All samples investigated in this study are made of carbon fiber composites. Two different materials were used. A list of samples and materials is presented in Table 1. Material A is a commercial pultruded composite plate (Sika CarboDur S1012) from Sika ${ }^{\circledR}$ group. This sample is composed of epoxy resin reinforced with uniaxial oriented carbon fibers.

Material B is a thermoplastic binder reinforced with woven carbon fibers, from Porcher Industries. The studied samples ratio of fibers in $X$ and $Y$ direction is equal to 3:1. Thus, two samples were cut along $X$ and $Y$ directions in order to study the influence of fiber density on thermophysical properties. Finally, for material B only, two sample thicknesses were considered. For the thicker sample, we considered only properties in $X$ direction. A sketch of the arrangement of fibers in both materials $A$ and $B$ is presented in figure 1.
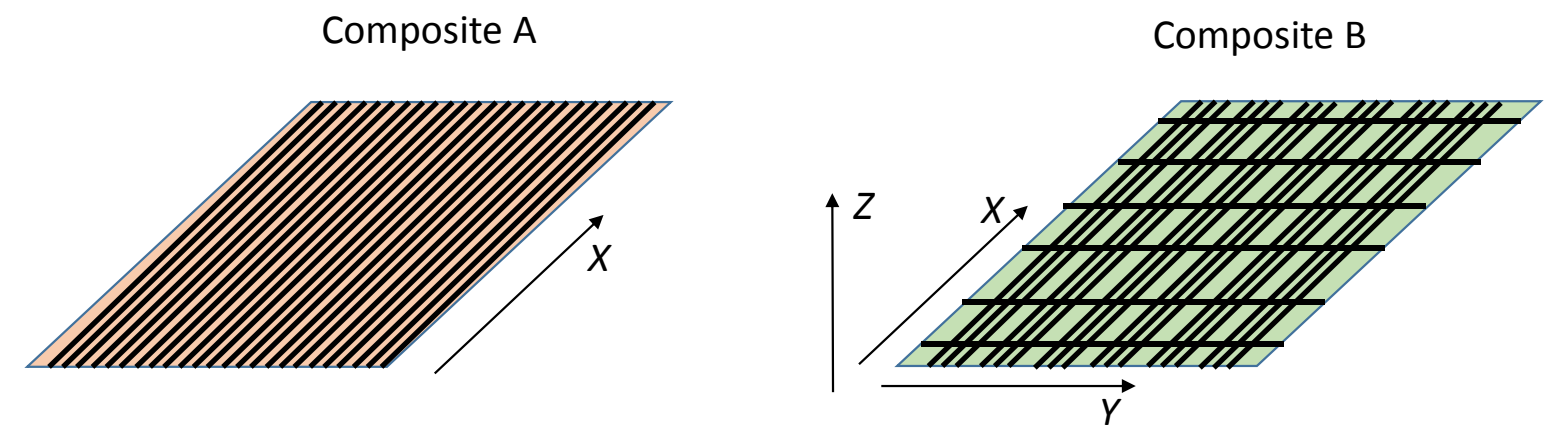

Fig. 1. Schematic representation of carbon fibers arrangement in materials $A$ and $B$ and directions of measurement 
http://dx.doi.org/10.21611/qirt.2014.204

Table 1. Carbon fiber reinforced composites samples studied

\begin{tabular}{|l|c|c|c|c|}
\hline Material Composition & $\begin{array}{c}\text { Uniaxial pultruded plate carbon } \\
\text { fibers / Epoxy resin }\end{array}$ & \multicolumn{3}{|c|}{$\begin{array}{c}\text { Bidirectional woven plate } \\
\text { carbon fibers / thermoplastic }\end{array}$} \\
\hline Material Ref / Fibers direction & $\mathrm{A} / \mathrm{X}$ & $\mathrm{B} / \mathrm{X}$ & $\mathrm{B} / \mathrm{X}$ & $\mathrm{B} / \mathrm{Y}$ \\
\hline Sample Thickness $(\mathrm{mm})$ & 1.36 & 1.85 & 2.55 & 1.85 \\
\hline Material density $\left(\mathrm{kg} / \mathrm{m}^{3}\right)$ & 1610 & 1400 & 1420 & 1410 \\
\hline
\end{tabular}

\subsection{Experimental set-up and raw measurements}

The experimental device developed is presented in figure 2. It was based on a preceding set-up devoted to emissivity measurement and estimation of thermal conductivity and diffusivity of isotropic materials (see refs [5,6] for details). The existing set-up and measurement protocol were modified in order to be able to determine in-plane thermal conductivity and diffusivity of carbon fibers composite samples. The sample holder is composed of a $10 \mathrm{~mm}$-thick Duralumin plate fixed onto a Thermoelectric cooler. The surface of the duralumin plate is of $44 \mathrm{~mm} \times 44 \mathrm{~mm}$. Composite plate samples can be fixed onto the sample holder. A thermally conductive grease can be used if necessary to reduce the thermal contact resistance between the Duralumin plate (sample holder surface) and the material to characterize. Sample plates are fixed onto the sample holder by using four screws. All samples used in this study have a length ranging between 150 and $200 \mathrm{~mm}$, a width of $40 \mathrm{~mm}$ and a thickness between ranging 1 and $3 \mathrm{~mm}$.

A sinusoidal current is imposed throughout the thermoelectric cooler in order to obtain a temperature modulation of the sample holder. To reduce experiment duration, a sum of five sinusoidal signals at five frequencies is used as excitation signal. Frequency values $f_{\mathrm{i}}$ are multiples of the fundamental frequency $f_{0}$ with $f_{\mathrm{i}}=2^{\mathrm{i}} \times f_{0}$. Sample holder temperature is measured using a K-type thermocouple inserted in the center of the Duralumin plate. As we will see later, this temperature is not used for the determination of thermophysical properties in $X$ and $Y$ directions.

The temperature of the composite plate is monitored using a long wave cooled FLIR SC7300 ${ }^{\mathrm{TM}}$ infrared camera in windowing mode. Excitation device, temperature measurements and image recordings are synchronised using a LabView $^{\mathrm{TM}}$ application. Figure 3 shows some infrared images of the sample A (pultruded composite plate) during measurements.

We can distinguish the part in contact with the sample holder and the left part of the composite plate that acts like a fin. For increase of the spatial resolution and symmetry reasons, only the left part of the composite plate was observed by the IR camera. Temperature values plotted are apparent temperatures. Indeed, the exact value of the absolute temperature and even of temperature variations are not required. This will be shown in next section.

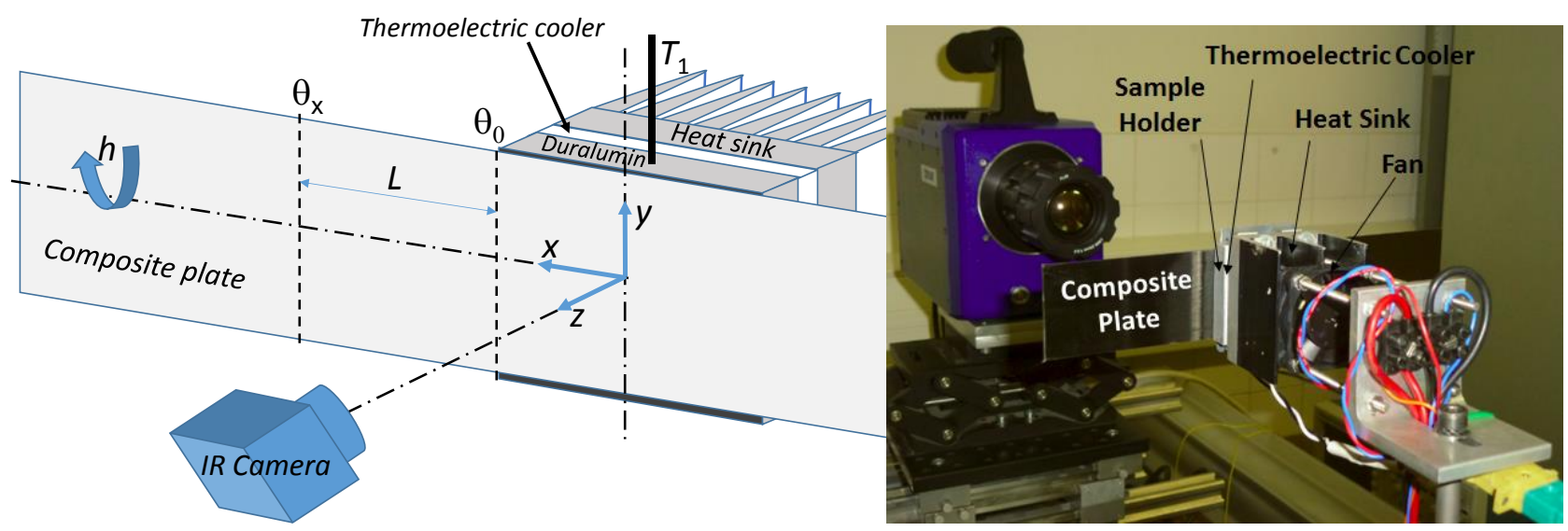

Fig. 2. View of the experimental set-up

\subsection{Heat transfer model and determination of in-plane thermal conductivity and diffusivity}

Heat transfer inside the composite plate is considered 1D in $x$-direction since the plate can be considered as a semi-infinite fin in this direction (Biot number is low in $z$-direction and infrared measurements show very low change of 
temperature according to $y$-direction). The solution of transient heat equation in periodic steady state and Laplace space is then given as follows [7]:

$$
\tilde{\theta}_{x}=\tilde{\theta}_{0} \exp (-\alpha x) \text { with } \alpha^{2}=\frac{h m}{k_{x} S}+\frac{p}{a_{x}}
$$

where $a_{x}$ is the thermal diffusivity in the $x$-direction, $k_{x}$ its thermal conductivity, $h$ the global heat transfer coefficient, $S$ the fin section and $m$ its perimeter. This can be re-written in the form:

$$
\frac{\tilde{\theta}_{x}}{\widetilde{\theta}_{0}}=M \exp (-j \Phi) \quad \text { with } \quad\left\{\begin{array}{l}
\ln M=-\beta_{M} x \\
\Phi=-\beta_{\Phi} x
\end{array}\right.
$$

The parameters $\beta_{M}$ and $\beta_{\Phi}$ are obtained by linear regression by plotting In $M$ and $\Phi$ according to $x$ for a given pulsation value $\omega$. This allows obtaining:

$$
\left\{\begin{array}{l}
a_{\mathrm{x}}=\frac{\omega}{2 \beta_{\mathrm{M}} \beta_{\Phi}} \\
\frac{h}{k_{\mathrm{x}}}=\frac{S}{m}\left(\beta_{\mathrm{M}}^{2}-\beta_{\Phi}^{2}\right)
\end{array}\right.
$$

For each pulsation $\omega$, the in-plane thermal diffusivity $a_{x}$ value can be directly computed from the slope values $\beta_{\mathrm{M}}$ and $\beta_{\Phi}$. However, the value of thermal conductivity $k_{x}$ can be determined only if the heat exchange coefficient $h$ value is known. This could be done by performing an independent experiment in the same conditions using a reference material and by considering that this parameter value does not depend on sample properties if experimental conditions and sample dimensions are similar. Such experiments are in progress, but results will not be presented in this paper.

Equations (2) and (3) show that the important information required for samples characterization are here the attenuation of temperature variations and the temperature phase shift along the sample plate. Sample temperature values are not required and thus the knowledge of the material emissivity is not needed.

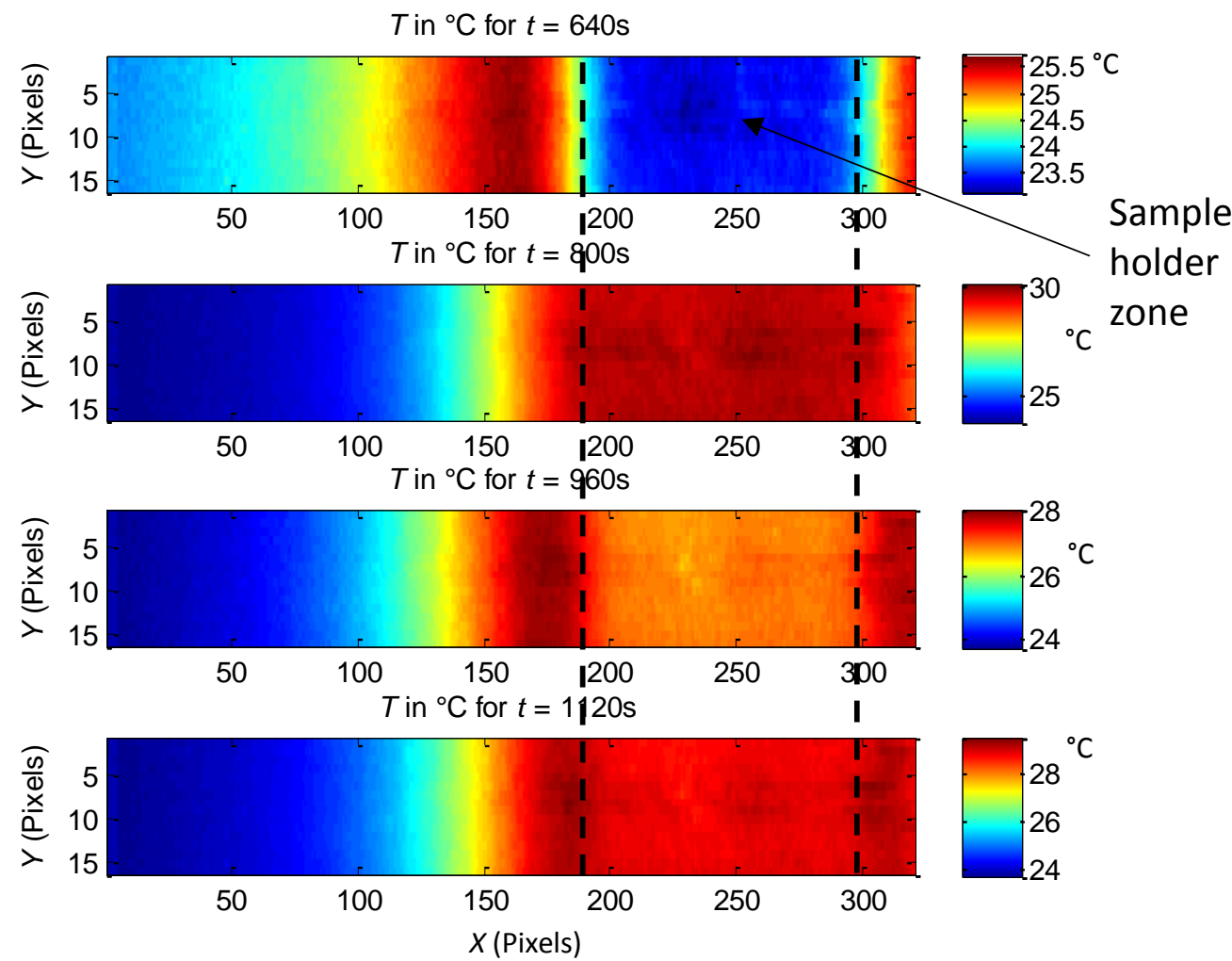

Fig. 3. View of some thermal images at different excitation times for sample $A$ 


\section{Results}

\subsection{Characterization of composite including carbon fibers with unidirectional orientation}

\subsubsection{Data processing}

The first numerical treatment performed is to compute an average temperature value along $y$-axis in the center part of the sample plate at each measurement time step. This allows obtaining a map presented in figure 4 , where the temperature is plotted as a function of time $t$ and position $L$.

The evolution of temperature at positions labelled $\theta_{0}$ and $\theta_{1}$ is presented in figure 5 (left part). For each position, a Fast Fourier Transform of the temperature vector is performed. The modulus of the FFT obtained for $\theta_{0}$ and $\theta_{1}$ positions is presented in Figure 5 (right part). $\theta 0$ position corresponds to the reference position, i.e. position of maximum of amplitude and null phase.

The value of the FFT at each excitation frequency allows then computing the attenuation and phase shift of temperature variations. An example of temperature attenuation and phase shit as a function of position $L$ is presented in Figure 6, for a sample of material A. A simple linear regression performed for each curve corresponding to one particular excitation frequency allows obtaining slope values $\beta_{\mathrm{M}}$ and $\beta_{\Phi}$. Finally, the use of Equation (3) allows computing values of in-plane thermal diffusivity and ratio between heat exchange coefficient and in-plane thermal conductivity.

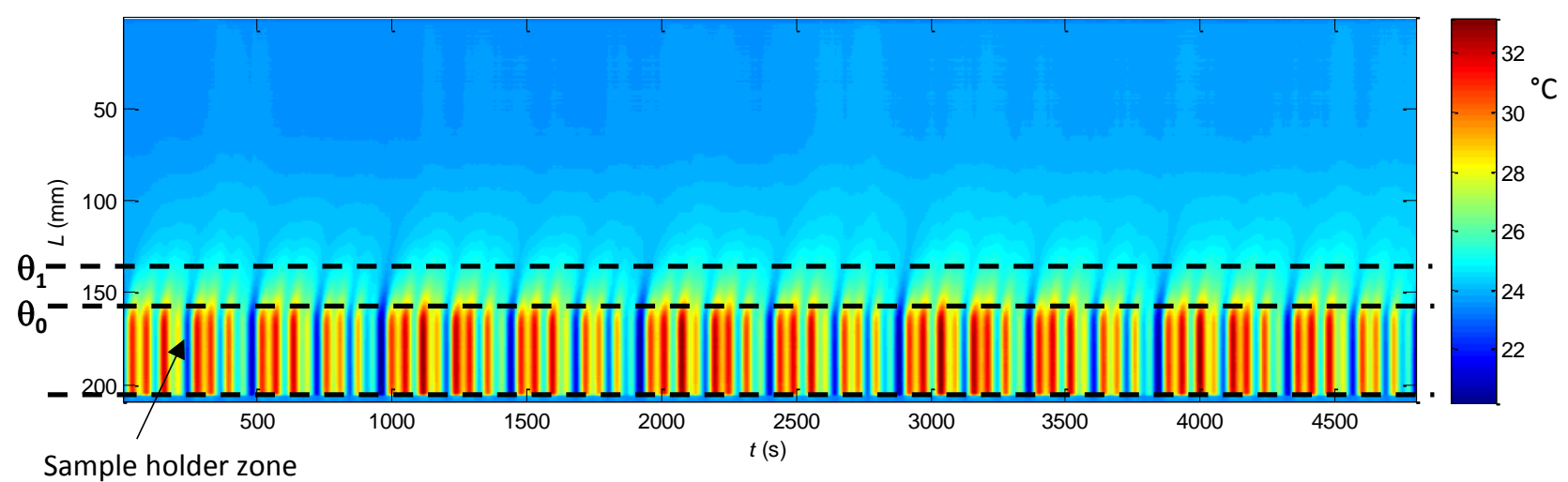

Fig. 4. Evolution of measured temperature along a sample of material A during an experiment with an excitation period of 960 s (total of five consecutive periods)

\subsubsection{Results}

Estimations results obtained for composite A are reported in Table 2 for an experiment performed using an excitation signal period of 640 s and a total amount of 5 periods. Thermal diffusivity obtained is typical for this kind of composite and is higher than the value obtained in the transverse direction of the composite plate $\left(a_{z}=0.25 \mathrm{~mm} / \mathrm{s}\right)$ using an independent characterization method [8]. The dispersion of thermal diffusivity values is lower than $9 \%$. However, a more important dispersion is obtained for the ratio $h / k(20 \%)$.

In order to check the reproducibility of measurements, the experiment was repeated after removing and replacing the sample (measurement $\mathrm{N}^{\circ} 2$ ). Besides, another experiment with different excitation signal frequencies was also performed (measurement $N^{\circ} 3$ ). Estimated values of $a_{x}$ and $h / k_{x}$ are presented in Table 3 . Results show a quite good reproducibility of results. Moreover, as expected, increasing the excitation signal period tends to reduce the dispersion of results.

Table 2. Estimations results for material $A$ (X direction) for an excitation signal period of 640 s

\begin{tabular}{|l|c|c|c|c|c|c|}
\hline Frequency $(\mathrm{mHz})$ & 1.56 & 3.12 & 6.25 & 12.5 & 25.0 & Mean values (standard deviation) \\
\hline Longitudinal diffusivity $\mathrm{ax}_{\mathrm{x}}\left(\mathrm{mm}^{2} / \mathrm{s}\right)$ & 5.07 & 4.90 & 4.18 & 4.29 & 4.31 & $4.55(0.40)$ \\
\hline Ratio $\mathrm{h} / \mathrm{k}_{\mathrm{x}}\left(\mathrm{m}^{-1}\right)$ & 1.56 & 1.60 & 1.63 & 2.13 & 2.33 & $1.85(0.36)$ \\
\hline
\end{tabular}


http://dx.doi.org/10.21611/qirt.2014.204

Table 3. Comparison of properties obtained from several measurements for material $A$ ( $X$ direction)

\begin{tabular}{|l|c|c|c|c|}
\hline Measurement $\mathrm{N}^{\circ}$ & 1 & $2^{\#}$ & $3^{\#}$ & \multirow{2}{*}{ Mean values } \\
\hline Excitation signal period (s) & 640 & 640 & 960 & 5 \\
\cline { 1 - 4 } Number of periods & 5 & 5 & 5 & 4.45 \\
\hline Longitudinal diffusivity $\mathrm{ax}_{\mathrm{n}}\left(\mathrm{mm}^{2} / \mathrm{s}\right)$ & $4.55(0.40)$ & $4.36(0.30)$ & $4.45(0.05)$ & 1.89 \\
\hline Ratio $\mathrm{h} / \mathrm{k}_{\mathrm{x}}\left(\mathrm{m}^{-1}\right)$ & $1.85(0.36)$ & $2.06(0.84)$ & $1.75(0.44)$ & \\
\hline
\end{tabular}

${ }^{\#}$ measurements performed after removing and replacing the sample
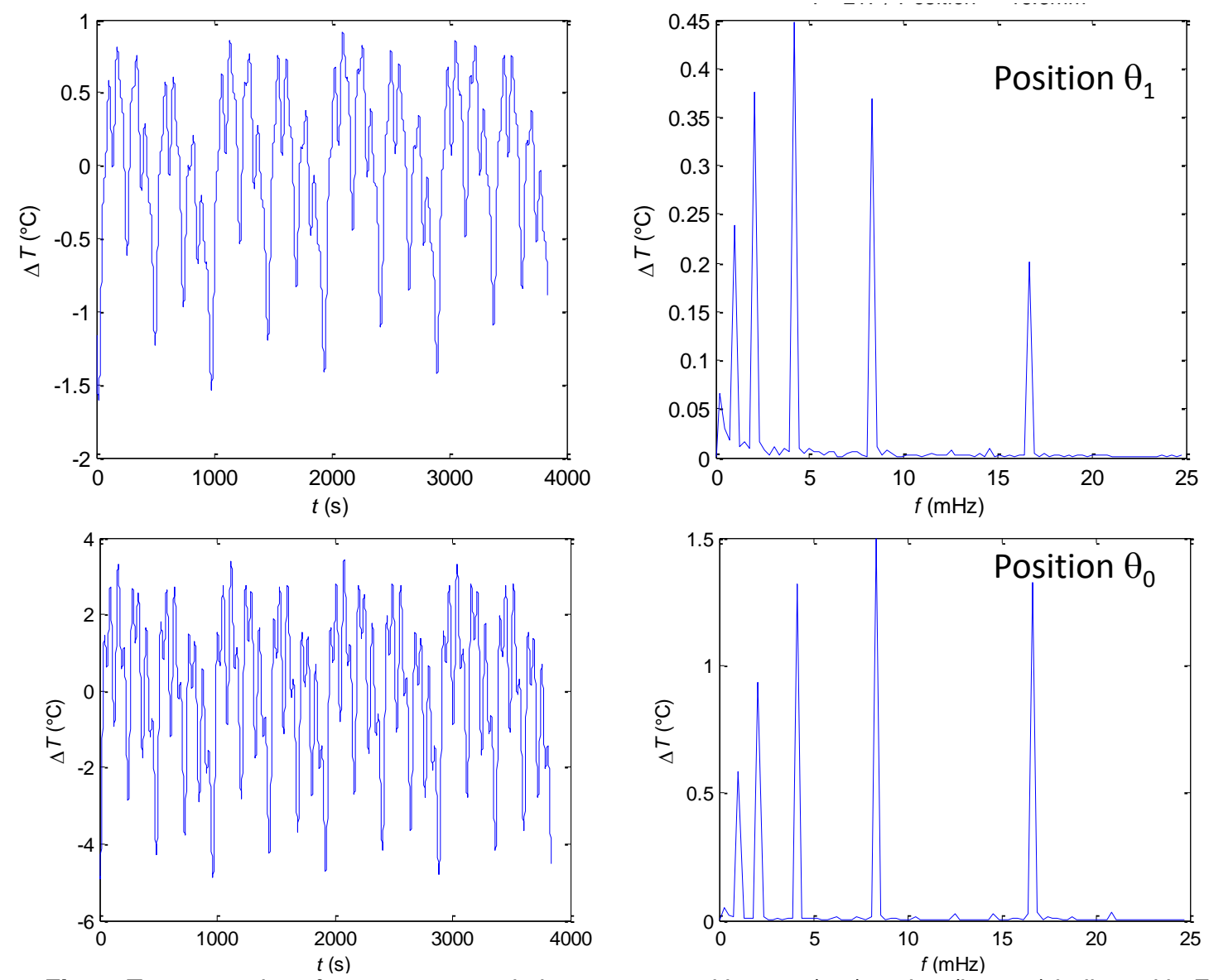

Fig. 5. Two examples of temperature variations at two positions $\theta_{1}$ (top) and $\theta_{0}$ (bottom) indicated in Figure 4 (sample of material A); raw data (left); Fast Fourier Transform (right)

\subsection{Effects of a bidirectional orientation of fibers and of sample thickness}

The effect of sample thickness and of a bidirectional orientation of fibers was investigated using material $\mathrm{B}$. Results obtained for the three samples characterized are presented in Table 4. Results show that the thickness of sample does not have any significant influence on the measured values of the thermal diffusivity. For the ratio $h / k$, highly dispersed values are obtained with this material compared to material A. It should be verified if this could be linked to the fact that material $B$ is less conducting than material $A$.

The ratio of thermal diffusivity values in $X$ and $Y$ directions ( $\left.a x / a_{Y}\right)$ is almost equal to 3 . This value corresponds to the ratio between fibers density along $X$ and $Y$ direction. If we compute the ratio of thermal conductivity in $X$ and $Y$ directions $(\mathrm{kx} / \mathrm{ky})$, by considering that $h$ value is constant from one experiment to another, we obtain a value of 2.7 which is also close to the expected value of 3 . 
The thermal diffusivity in $Z$ direction was measured by an independent method [8]. A value of about $0.5 \mathrm{~mm}^{2} / \mathrm{s}$ was found. As observed for material $A$ and as expected, the in-plane thermal diffusivity is higher than the transverse one: here diffusivity ratios of $a x / a z=6$ and $a y / a z=2$ are observed.
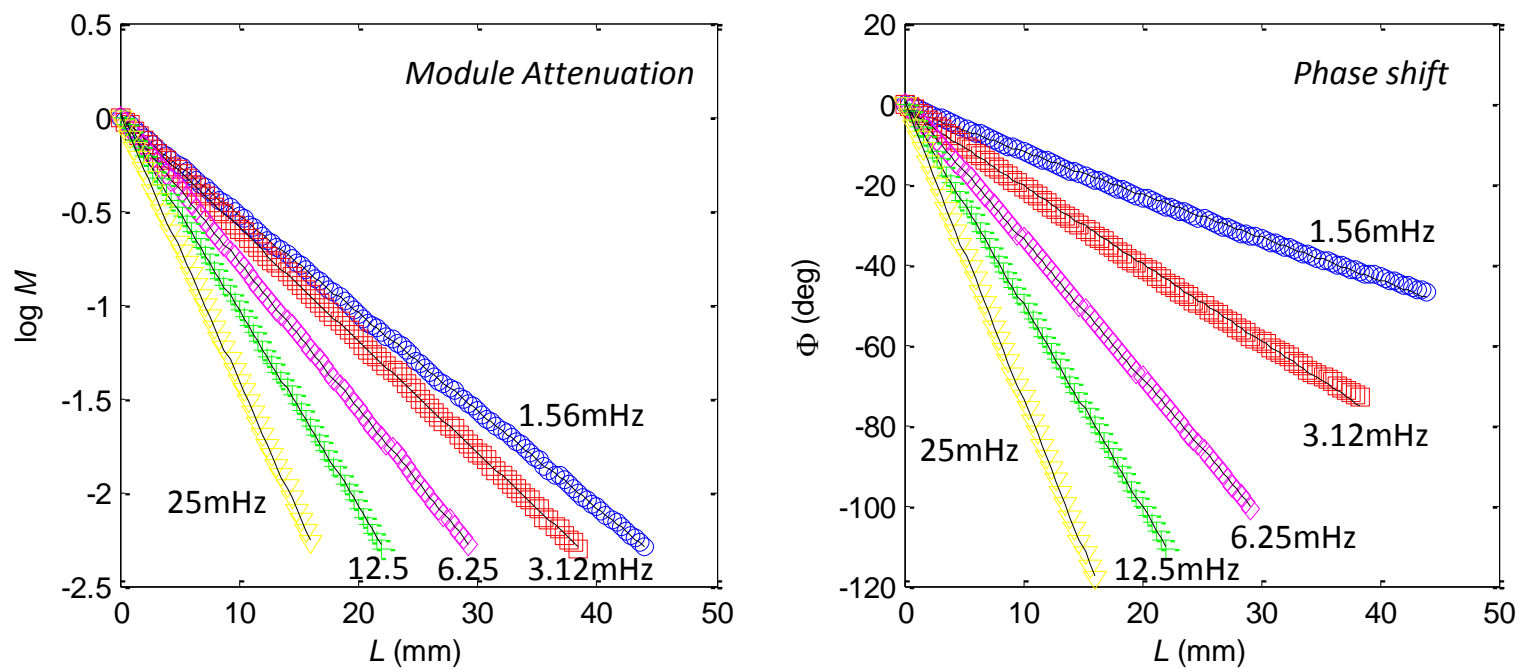

Fig. 6. Comparison of experimental (symbols) and theoretical (lines) module attenuation and phase shift along the composite fin position L; plots for five excitation frequencies (sample of material A)

Table 4. Comparison of properties obtained from several samples of material $B$

\begin{tabular}{|l|c|c|c|}
\hline Material / Direction & $\mathrm{B} / \mathrm{X}$ & $\mathrm{B} / \mathrm{X}$ & $\mathrm{B} / \mathrm{Y}$ \\
\hline Thickness $(\mathrm{mm})$ & 1.85 & 2.55 & 1.85 \\
\hline Excitation signal period $(\mathrm{s})$ & 640 & 1024 & 1024 \\
\hline Number of periods & 5 & 5 & 5 \\
\hline Longitudinal diffusivity $a_{x / y}\left(\mathrm{~mm}^{2} / \mathrm{s}\right)$ & $3.02(0.16)$ & $2.98(0.10)$ & $1.00(0.03)$ \\
\hline Ratio $h / k_{\mathrm{x} / \mathrm{y}}\left(\mathrm{m}^{-1}\right)$ & $4.04(2.9)$ & $3.54(2.3)$ & $11.0(5.3)$ \\
\hline
\end{tabular}

\section{Conclusion}

This work was devoted to the development of a new device for the determination of thermal conductivity and diffusivity of anisotropic composite plates. The excitation signal is provided through a thermoelectric cooler and does not require any optical source like a laser source for instance. The in-plane thermal diffusivity can be measured without an exact knowledge of sample emissivity. Sample plates of thicknesses up to $2.5 \mathrm{~mm}$ can be characterized. Determination of the thermal conductivity requires a calibration of the device with a reference sample.

Forthcoming work will first concern the validation of the calibration procedure for the determination of the thermal conductivity, the characterization of other types of anisotropic materials, the determination of the measurement limits of the method. A particular attention will be paid to the evaluation of uncertainties of measured values of thermal conductivity and diffusivity and to the possible ways to reduce measurement uncertainties. Finally, a possible determination of thermophysical properties in $Z$ direction (i.e. perpendicular to the sample plate) is also investigated. Nevertheless, in that case, emissivity value of the sample will be required.

\section{REFERENCES}

[1] $\mathrm{ACl}$ Committee 440.2R02-08, "Guide for the Design and Construction of Externally Bonded Systems for Strengthening Concrete Structures", American Concrete Institute, Michigan, U.S.A., 2008.

[2] Dumoulin J., Taillade F., Aubagnac Ch., Benzarti K. and Quiertant M., Infrared Thermography for the Nondestructive Inspection of CFRP Strenghtening, Concrete International, vol. 33, issue 4, April 2011, pp. 5458.

[3] Crinière A., Dumoulin J., Ibarra-Castanedo C. and Maldague X., Inverse model for defect characterization of externally glued CFRP on reinforced concrete structures: Comparative study of square pulsed and pulsed 
thermography, Quantitative InfraRed Thermography Journal, Taylor \& Francis Editor, 2014. DOI: 10.1080/17686733.2014.897512.

[4] Feuillet V., Ibos L., Fois M., Dumoulin J., Candau Y., "Defect detection and characterization in composite materials using square pulse thermography coupled with singular value decomposition analysis and thermal quadrupole modeling", NDT \& E International, vol. 51, pp. 58-67, 2012

[5] Datcu S., Ibos L., Candau Y., Mattéï S., "Improvement of building surface temperature measurements by infrared thermography". Infrared Physics and Technology, vol. 46, pp. 451-467, 2005.

[6] Agoudjil B.,.Datcu S., Boudenne A., Ibos L., Candau Y., "Parametric estimation of thermoradiative properties of materials based on harmonic excitation". Review of Scientific Instruments, vol. 77, pp. 035106-1 to 9, 2006.

[7] Degiovanni A., "Transmission de l'énergie thermique - conduction", Techniques de l'ingénieur, BE 8 200, pp. 125, 1999.

[8] Boudenne A., Ibos L., Géhin E., Candau Y., "A simultaneous characterisation of thermal conductivity and diffusivity of polymer materials by a periodic method", Journal of Physics D : Applied Physics, vol. 37(1), pp. 132-139, 2004 